\title{
Azote, rendement et qualité des graines : mise au point et utilisation du modèle Azodyn-colza pour améliorer les performances du colza vis-à-vis de l'azote
}

\author{
Marie-Hélène JEUFFROY ${ }^{1}$ \\ Muriel VALANTIN-MORISON ${ }^{1}$ \\ LUC CHAMPOLIVIER ${ }^{2}$ \\ Raymond REAU ${ }^{1,2}$ \\ ${ }^{1}$ INRA, UMR Agronomie (211) \\ INRA-AgroParisTech, BP01, \\ 78850 Thiverval-Grignon, France \\ ${ }^{2}$ CETIOM, BP4, \\ 78850 Thiverval-Grignon, France
}

\begin{abstract}
Nitrogen management is complex in oilseed-rape for several reasons: the crop can uptake high amounts of nitrogen during autumn, but a part of this nitrogen is lost through leaves fall during winter and spring, event if these fallen leaves are mineralized and contribute partly to the crop nitrogen nutrition. Moreover, recent results have shown that some periods of $N$ deficiency during the crop cycle are not detrimental for yield. Finally, higher seed oil content are obtained with lower nitrogen fertilisation. All these results suggest that a sub-optimal nitrogen nutrition of the crop could lead to high yields and high seed quality, with low losses through environment. In order to design such fertilisation strategies, the use of a crop model, simulating the variables determinant in the yield and grain quality formation and their interaction with environment, can help advisors. As numerous results on the yield and quality determinants of a rape crop have been obtained in the recent years, the available Azodyn-wheat model has been adapted to the specificities of this crop, based on this knowledge. This paper aims at describing the main results on yield and quality elaboration of a rape crop, and then the designing of the Azodyn-rape model. Then, some results of the model evaluation are given and uses of the model for nitrogen fertilisation management are proposed.
\end{abstract}

Key words: oilseed rape, nitrogen, yield formation, seed quality, fertilisation strategies

en cause ces pratiques d'assurance pour tenter de maximiser l'efficience d'utilisation de l'azote apporté et de réduire les pertes. De plus, l'intérêt économique de stratégies de fertilisation azotée suboptimales a été largement démontré sur blé $[4,5]$. Or, des résultats récents sur les conséquences de carences azotées chez le colza selon leur période d'occurrence ont montré que certaines carences ne conduisent pas à des réductions de rendement [6]. Par ailleurs, la fertilisation azotée a un effet non négligeable sur la qualité des graines récoltées: plus la culture est fertilisée, moins les graines sont riches en huile [7]. Or ce critère intervient dans la rémunération des graines aux organismes stockeurs. Des travaux récents sur tournesol [8] ont montré qu'une adaptation de la conduite de culture, et notamment une maîtrise de la fertilisation azotée, offre des perspectives réelles d'amélioration des résultats de qualité et de rentabilité pour les agriculteurs et les organismes de collecte. Aujourd'hui, le développement de l'utilisation du colza pour la fabrication de biocarburants permet d'accroître substantiellement l'offre de tourteaux, dont la richesse en protéines intéresse les fabricants d'aliments du bétail. Or, de nombreux auteurs $[7,9,10]$ ont démontré qu'il existait une relation négative entre la teneur en huile et la teneur en protéines des graines de colza.

Pour améliorer la gestion de la fertilisation azotée, dans le but de permettre un rendement élevé et une qualité des graines optimale pour les divers utilisateurs, et de limiter les impacts environnementaux, il paraît donc intéressant de disposer d'outils de simulation reposant sur la quantification des processus déterminants dans l'élaboration du rendement et de la qualité des graines et de leurs interactions durant le cycle cultural de la culture. Pour être efficaces, ces outils doivent rendre compte des effets des facteurs de croissance les plus importants rencontrés dans les conditions de culture du colza, notamment la température, le rayonnement, et l'eau, en considérant, dans un premier temps, que les bioagresseurs peuvent être évités ou éliminés.

Le rendement du colza se fabrique à travers la mise en place de différents organes, tout au long du cycle: ramifications, inflorescences, siliques, graines. Cette plante ayant un développement indéterminé, les étapes de mise en place de ces différents organes se chevauchent dans le temps et rendent particulièrement difficile l'étude de leur déterminisme et leur quantification [11]. Plusieurs modèles de culture rendent compte de ces étapes successives et de l'élaboration de ces différentes composantes, mais ils reposent sur un grand nombre de relations et de paramètres et sont de fait très sensibles à des erreurs d'estimation de ces paramètres $[12,13]$.

Sur le blé, un modèle plus simple a été mis au point $[14,15]$ et utilisé pour raisonner la fertilisation azotée de la culture, en agriculture conventionnelle [16] ou en agriculture biologique [17], en vue de maximiser le revenu économique et la qualité des graines et de minimi- 
ser les pertes azotées vers l'environnement. Depuis 2000, des travaux ont permis d'adapter ce modèle à la culture du colza, en tenant compte des spécificités de cette culture, notamment grâce à l'acquisition de nombreuses connaissances sur son fonctionnement dans différentes conditions de culture.

Cet article vise, dans une première partie, à synthétiser ces connaissances qui ont permis de progresser dans la compréhension de l'élaboration du rendement et de la qualité de cette culture. Dans la seconde partie, nous montrerons comment ces connaissances ont été intégrées dans le modèle Azodyn-colza, simulant le rendement, la teneur en protéines et en huile des graines d'une culture de colza. Enfin, dans une dernière partie, nous préciserons les résultats d'évaluation du modèle et indiquerons quelques exemples actuels et futurs de son utilisation en vue d'améliorer la gestion de la fertilisation azotée du colza.

\section{Processus en jeu dans l'élaboration du rendement et de la qualité des graines de colza}

\section{La floraison : un moment clef pour construire le nombre de graines}

Comme chez de nombreuses espèces, le rendement du colza est étroitement corrélé au nombre de graines formées par $\mathrm{m}^{2}$. Le nombre de fleurs à l'origine des graines est très variable et explique une part importante de la variabilité du nombre de graines. II est étroitement dépendant du nombre d'inflorescences mises en place [18]. La période de formation des graines est extrêmement variable chez le colza. Elle dépend de la durée pendant laquelle une plante émet de nouvelles fleurs, liée au développement indéterminé de la plante, et à la présence d'un nombre variable de ramifications, dont la floraison n'est pas synchrone avec celle de l'inflorescence principale. Une partie variable des fleurs, ainsi que des graines dans les siliques, peuvent avorter sur une plante, conduisant à une variabilité importante du nombre de siliques par plante et du nombre moyen de graines par silique. Tayo et Morgan [19] ont montré que $75 \%$ des siliques présentes à la récolte, sur une plante, proviennent des fleurs produites pendant les onze premiers jours de la floraison.

Selon Leterme [11], à l'échelle d'une silique, la formation des graines, concomitante de la croissance en longueur de la silique, dure 300 degrés-jours. Pendant cette phase, le nombre de graines formées à l'intérieur d'une silique dépend de la quantité d'assimilats carbonés alloués à cette silique.

\section{La période de remplissage des graines}

À partir du moment où les graines sont formées, elles commencent à accumuler différents types d'éléments (lipides, protéines, sucres, etc.). La période de remplissage des différentes graines de la plante n'est donc pas synchrone. Chez le blé et le pois, un modèle dynamique simulant les flux d'azote et de carbone vers les grains en fonction de leur capacité d'accumulation et de la disponibilité de ces éléments sur la plante a été mis au point $[20,21]$. Les connaissances sur la dynamique d'accumulation de la biomasse, des protéines et de l'huile dans les graines et sur l'effet du stress hydrique sur les processus de remplissage des graines sont très réduites sur colza, ce qui a conduit à faire des hypothèses de modélisation fortes pour simuler la teneur en huile.

\section{L'évolution de l'indice foliaire et de la biomasse verte}

L'évolution de l'indice foliaire d'une culture de colza est la résultante du processus d'émission et de croissance des feuilles mais aussi du processus de chute des feuilles, qui réduit progressivement la surface des feuilles interceptrices. La durée de vie des feuilles a été estimée à 650 degrés-jours [22]. Chez de nombreuses espèces (blé, tomate, pois, laitue, graminées fourragères), une relation linéaire entre l'indice foliaire et la quantité $d^{\prime} N$ accumulée par la culture à son optimum de nutrition azotée a été mise en évidence [23]. Une telle relation a également été observée chez le colza [24]. La croissance aérienne de la culture est fonction du rayonnement intercepté par la culture (luimême fonction de son indice foliaire) et de I'efficience de conversion du rayonnement en biomasse. Cette efficience est variable en fonction de la température et de l'état de nutrition azotée de la culture [25] et peut être réduite en cas de contrainte hydrique.

\section{L'accumulation de l'azote et les effets des carences azotées}

L'accumulation d'azote par une culture de colza est limitée par la disponibilité en azote minéral dans le sol et par la capacité d'absorption de la culture [26]. Cette dernière peut être quantifiée grâce à l'évolution de la teneur en $\mathrm{N}$ maximale des parties aériennes de la culture, définie par Colnenne et al. [27], et de l'évolution de la biomasse aérienne de la culture.

L'N minéral du sol, utilisable par la culture, peut provenir (i) de la minéralisation nette de I'humus, des résidus de récolte du précédent cultural et des amendements organiques apportés antérieurement sur la parcelle, (ii) de la minéralisation des feuilles mortes tombées au cours du cycle cultural, (iii) de l'engrais azoté apporté sur la culture. La minéralisation des feuilles mortes est liée à leur contenu en azote, lui-même fonction du statut azoté de la culture au moment de leur chute [22]. Dans certaines conditions, la culture peut donc perdre instantanément une quantité importante d'azote. Une partie seulement de l'azote issu de la minéralisation des feuilles mortes est réutilisée par la culture (environ $40 \%$ ), une partie non négligeable étant perdue par volatilisation [28]. L'efficience d'utilisation de l'azote issu des engrais minéraux apportés est très variable, fonction des conditions climatiques au moment de l'apport mais aussi des besoins instantanés de la culture à absorber, comme I'ont montré Limaux et al. [3] sur blé. La relation entre le coefficient apparent d'utilisation de l'azote et la vitesse de croissance de la culture au moment de l'apport d'engrais, mise au point par ces auteurs, a été paramétrée sur colza. Cette relation est moins précise que celle observée sur blé, du fait de la difficulté à tenir compte de l'azote accumulé par la culture mais perdue par la chute continue des feuilles, rendant difficile la quantification de l'accumulation maximale d'azote par la culture, et de l'identification peu précise du stade auquel l'accumulation d'azote semble être stoppée. Faure [29] a montré que le développement de l'appareil reproducteur entraîne chez le colza un effondrement des capacités d'absorption du nitrate. Sur la base de ces travaux, la date de l'arrêt de l'absorption de l'azote par la culture a été identifiée au stade fin floraison.

Si la culture subit une période de carence azotée pendant son cycle, le nombre de graines peut être réduit [6]. Cependant, dans la majorité des situations, les carences automnales ne semblent pas d'avoir d'effet majeur sur le rendement [30, 31]. Jeuffroy et al. (2003) ont montré que la perte de nombre de graines était majoritairement liée à l'état de nutrition azotée de la culture entre $\mathrm{F} 1$ et $\mathrm{G} 4$. En présence d'une carence azotée, I'indice foliaire et l'efficience de conversion du rayonnement en biomasse sont réduits, la réduction étant fonction du statut azoté de la culture, caractérisé à travers son indice de nutrition azotée [30]. Ces effets induisent une réduction de l'accumulation de biomasse par la culture.

\section{Intégrer ces connaissances dans le modèle Azodyn-colza}

\section{Description générale du modèle}

Toutes ces connaissances ont été intégrées dans un modèle dynamique à pas de temps journalier simulant la croissance aérienne, l'accumulation d' $\mathrm{N}$ par la culture, l'élaboration du rendement et la qualité des graines d'une culture de colza d'hiver. Par rapport au modèle 
Azodyn-blé, dont il est issu, le modèle Azodyncolza comporte un module supplémentaire simulant la chute des feuilles et leur devenir. De même, un module hydrique simulant les transferts d'eau (et de l'azote) dans le sol et la culture, ainsi que leurs conséquences sur la disponibilité d'azote dans le sol et la croissance de la culture, a été inclus dans le modèle. Le module hydrique n'avait pas été intégré initialement dans le modèle blé, mais il a été considéré nécessaire dans le modèle colza, du fait que cette culture est fréquemment cultivée sur des petites terres, dans des régions où la disponibilité en eau est limitante pour la culture. De même, un module simulant le comportement de la culture pendant l'automne et l'hiver a été introduit afin de simuler la variabilité de l'absorption de l'azote pendant l'automne, la chute des feuilles et les risques de lixiviation du nitrate pendant l'hiver. Contrairement à Azodyn-blé, pour lequel les simulations commencent à l'ouverture du bilan azoté (sortie hiver), les simulations commencent, dans le modèle colza, dès la date de récolte de la culture précédant le colza, à partir de la connaissance du contenu en eau et en azote du sol et de la quantité de résidus du précédent.

Finalement, le modèle comporte un module simulant la croissance de la culture, l'évolution de son indice foliaire et son accumulation d'azote, un module sol, décrivant la disponibilité de l'azote minéral dans le sol et les transferts d'eau dans le sol, et un module simulant l'élaboration du rendement et la qualité des graines.

\section{Le module plante}

La croissance aérienne de la culture est simulée à partir du formalisme de Monteith (fonction du rayonnement intercepté), choisi pour son faible nombre de paramètres et leur disponibilité dans la littérature. La simulation de la biomasse et du contenu en $N$ des racines est réalisée à partir de ratios partie aérienne/partie racinaire. L'évolution de l'indice foliaire, hors contrainte azotée et hydrique, dépend de l'azote accumulé par la culture. Les effets de carences azotées sont simulés grâce au calcul de l'indice de nutrition azotée. L'INN (instantané ou intégré) a un effet à la fois sur l'indice foliaire, l'efficience de conversion du rayonnement en biomasse et le nombre de grains formés. La simulation de la biomasse et du contenu en $\mathrm{N}$ des racines est réalisée à partir de ratios partie aérienne/partie racinaire. La dynamique journalière de chute des feuilles, et la perte induite d'indice foliaire, de biomasse et d'azote dans la culture, sont simulées en fonction de la durée de vie des feuilles, comme décrit dans la partie précédente.

\section{Le module sol}

La disponibilité en eau du sol est simulée grâce à un modèle réservoir. Le module hydrique considère 3 couches de sol. La première correspond à la zone de sol explorée par les racines, dont la profondeur varie au cours du temps en fonction de la dynamique de croissance du système racinaire, elle-même calculée en fonction de la température aérienne à partir de la levée. La seconde couche de sol varie également au cours du cycle cultural. Elle est comprise entre la profondeur d'enracinement à la date considérée et la profondeur maximale d'enracinement de la culture, elle-même limitée soit par la culture (paramètre lié à l'espèce), soit par la profondeur du sol de la parcelle. Enfin, la dernière couche est celle située sous la profondeur maximale d'enracinement.

La disponibilité en azote dans le sol est simulée sur la base de la méthode du bilan [32], permettant un paramétrage simple en fonction des situations culturales en France, la plupart des paramètres étant déjà disponibles dans la littérature. La minéralisation de l'humus et des résidus dépend de la température, de l'humidité du sol et de caractéristiques intrinsèques à la parcelle. Comme décrit dans la première partie, la minéralisation des feuilles mortes est liée à leur contenu en azote, lui-même fonction du statut azoté de la culture au moment de leur chute [22]. Le modèle de lessivage du nitrate, proposé par Burns [33] (1976), choisi pour sa simplicité d'adaptation aux situations culturales, a été inclus dans le modèle.

\section{La simulation du rendement et de la qualité des graines}

D'après les résultats décrits dans la première partie de cet article, le nombre de graines potentiel de la culture a été déterminé en fonction du rayonnement intercepté par la culture pendant les 600 premiers degrés-jours de la floraison [34]. Ce nombre potentiel peut ensuite être réduit en fonction de l'indice de nutrition azotée de la culture entre F1 et G4. Ne disposant pas des connaissances nécessaires à la mise au point d'un modèle dynamique de l'accumulation d'azote dans les graines sur le colza, la simulation de la teneur en protéines et en huile des graines a été réalisée de manière statique et simplifiée. La corrélation étroite existant entre la teneur en huile et la teneur en protéines pour une variété donnée est intégrée dans le modèle [34]. Une relation entre l'état azoté de la culture au début floraison (INN à F1) et la teneur en protéines «potentielle » de la culture a été mise en évidence sur une large gamme de situations [34]. Cette teneur en protéines peut alors être modifiée selon les conditions de remplissage des graines, par l'intermédiaire de la variabilité du PMG, liée aux températures trop faibles ou trop élevées et au stress hydrique susceptibles de limiter le niveau de croissance des graines.

\section{Les entrées et les sorties}

Le modèle simule le rendement (et ses deux composantes, nombre de graines par $\mathrm{m}^{2}$ et poids moyen d'une graine), la teneur en huile et en protéines des graines à la récolte, la quantité $\mathrm{d}^{\prime} \mathrm{N}$ perdu par lessivage et par volatilisation, le reliquat $\mathrm{d}^{\prime} \mathrm{N}$ minéral dans le sol à la récolte et la quantité $\mathrm{d}^{\prime} \mathrm{N}$ dans les résidus de colza.

Ses entrées sont les modalités de certaines techniques (date et densité de semis, modalités de fertilisation azotée, apport d'amendements organiques, gestion des résidus du précédent), certains stades du colza (début floraison), les caractéristiques du sol (humidité au point de flétrissement permanent et à la capacité au champ, texture, contenu en $\mathrm{N}$ organique et en calcaire de la couche labourée, profondeur maximale d'enracinement), la quantité et la nature des résidus du précédent cultural, le climat journalier (température, rayonnement, ETP, pluviométrie) entre la date de récolte du précédent et la date de récolte du colza, et l'état du sol (eau et azote) à la date d'initialisation de la simulation. La plupart de ces entrées peuvent facilement être acquises en parcelles agricoles.

La majorité des paramètres est directement mesurable expérimentalement, ce qui permet d'adapter facilement le modèle à de nouvelles variétés, tout en conservant la signification biologique de ses paramètres.

\section{Évaluation du modèle et utilisations pour la gestion de la fertilisation azotée}

\section{Évaluation du modèle}

Plusieurs évaluations du modèle ont été réalisées en vue d'estimer sa qualité prédictive, dans une large gamme de dates de semis (de début juillet à début septembre), de densités de semis (de 25 à 150 plantes. $\mathrm{m}^{-2}$ ), et de fertilisation azotée (doses totales appliquées variant de 0 à $300 \mathrm{~kg} \cdot \mathrm{ha}^{-1}$ ) pendant plusieurs années. Dans ces situations, le rendement a varié de 15 à $39 \mathrm{~g} / \mathrm{ha}$, la teneur en protéines des graines de $16,3 \%$ à $25,8 \%$ et leur teneur en huile de $42,5 \%$ à 49,2\%. Dans ces conditions, la dynamique d'évolution de l'indice foliaire et de la biomasse aérienne a été bien simulée, l'erreur de prédiction (RMSE) étant respectivement de 1,00 et 1,82 t.ha $^{-1}$ [35]. En revanche, la prédiction de l'accumulation d'azote dans la plante était moins bonne, I'erreur de prédiction étant alors de 56,7 kg.ha- ${ }^{-1}$. 
Tableau 1. Conséquences de stratégies de fertilisation azotée, simulées avec Azodyn-colza dans une même parcelle semée le 25 août ( $1^{\text {er }}$ tableau) ou le 15 août (2e tableau), sur le coefficient apparent d'utilisation de l'azote de chaque apport (CAU, \%), le rendement et la teneur en huile des graines (d'après Valantin-Morison et al., 2004).

\begin{tabular}{|c|c|c|c|c|c|c|c|c|c|c|c|c|}
\hline \multirow[t]{2}{*}{ Stratégie } & \multicolumn{3}{|c|}{$1^{\text {er }}$ apport d'N } & \multicolumn{3}{|c|}{$2^{e}$ apport $d^{\prime} N$} & \multicolumn{3}{|c|}{$3^{e}$ apport $d^{\prime} N$} & \multirow[t]{2}{*}{ Dose totale } & \multirow[t]{2}{*}{ Rendement } & \multirow[t]{2}{*}{$\%$ huile } \\
\hline & date & dose & CAU & & & & & & & & & \\
\hline 1 & $30 / 01$ & 80 & 30 & $25 / 03$ & 150 & 82 & $15 / 04$ & 0 & & 230 & 29.9 & 47.2 \\
\hline 2 & $30 / 01$ & 40 & 30 & $25 / 03$ & 170 & 82 & $15 / 04$ & 0 & & 210 & 29.9 & 47.5 \\
\hline 3 & $30 / 01$ & 0 & & $25 / 03$ & 80 & 82 & $15 / 04$ & 150 & 100 & 230 & 31.2 & 48.0 \\
\hline 4 & $30 / 01$ & 0 & & $25 / 03$ & 40 & 82 & $15 / 04$ & 170 & 100 & 210 & 29.6 & 49.0 \\
\hline 5 & $7 / 03$ & 40 & 98 & $25 / 03$ & 70 & 82 & $15 / 04$ & 120 & 100 & 230 & 33.3 & 48.0 \\
\hline \multirow[t]{2}{*}{ Stratégie } & \multicolumn{3}{|c|}{$1^{\text {er }}$ apport d'N } & \multirow{2}{*}{\multicolumn{3}{|c|}{$2^{e}$ apport $d^{\prime} N$}} & \multirow{2}{*}{\multicolumn{3}{|c|}{$3^{e}$ apport d'N }} & \multirow[t]{2}{*}{ Dose totale } & \multirow[t]{2}{*}{ Rendement } & \multirow[t]{2}{*}{$\%$ huile } \\
\hline & date & dose & CAU & & & & & & & & & \\
\hline 6 & $30 / 01$ & 80 & 24 & $25 / 03$ & 150 & 73 & $15 / 04$ & 0 & & 230 & 31.3 & 46.1 \\
\hline 7 & $30 / 01$ & 40 & 24 & $25 / 03$ & 170 & 69 & $15 / 04$ & 0 & & 210 & 31.1 & 46.5 \\
\hline 8 & $30 / 01$ & 0 & & $25 / 03$ & 80 & 65 & $15 / 04$ & 150 & 100 & 230 & 32.7 & 46.9 \\
\hline 9 & $30 / 01$ & 0 & & $25 / 03$ & 40 & 65 & $15 / 04$ & 170 & 100 & 210 & 30.5 & 48.2 \\
\hline 10 & $7 / 03$ & 40 & 80 & $25 / 03$ & 70 & 75 & $15 / 04$ & 120 & 100 & 230 & 34.6 & 45.6 \\
\hline
\end{tabular}

Cet écart était principalement lié à une surestimation de l'accumulation d'azote à l'automne, dans les situations sans apport $d^{\prime} \mathrm{N}$. En revanche, dans les cas de fertilisation azotée, les simulations étaient correctes et le modèle rendait correctement compte des fortes absorptions observées en relation avec les fortes disponibilités d'azote minéral dans le sol. Au printemps, le modèle simulait correctement les périodes de carence azotée. À la récolte, les erreurs de prédiction étaient de 5.3 q.ha ${ }^{-1}$ pour le rendement, de 13920 graines par $\mathrm{m}^{2}$, de 0,34 mg par graine, de $3,1 \%$ pour la teneur en huile et de $4.5 \%$ pour la teneur en protéines des graines. Des évaluations dans une plus large gamme de contextes pédoclimatiques (4 régions contrastées en France) ont donné des résultats similaires [36].

\section{Utilisation du modèle pour raisonner la fertilisation azotée à l'échelle de la parcelle}

Le modèle a ensuite été utilisé pour simuler les conséquences de stratégies innovantes de fertilisation azotée, dans différentes situations variant par la disponibilité en $\mathrm{N}$ dans le sol et par la date de semis du colza $[35,36]$ (tableau 1). Pour une même dose totale appliquée, les stratégies conduisant aux meilleurs rendements comportaient un faible apport $d^{\prime} \mathrm{N}$ à la reprise de végétation (voire même un apport nul), et des doses plus élevées aux second et troisième apports. Ces stratégies permettent d'assurer des coefficients apparents d'utilisation (CAU) moyens plus élevés. Par ailleurs, dans les situations testées, une réduction de la dose totale appliquée a conduit à une augmentation systématique de la teneur en huile des graines et à une réduction systématique de la quantité $\mathrm{d}^{\prime} \mathrm{N}$ minéral restant dans le sol à la récolte du colza. Pour ces fertilisations réduites, dans des situations de colza relativement gros après hiver, le fractionnement optimal semble correspondre à une absence de $1^{\text {er }}$ apport à la reprise de végétation, et des fortes applications pouvant être reportées au $3^{\mathrm{e}}$ apport (tableau 1). De plus, à même dose d'azote apporté, le décalage du premier apport $d^{\prime}$ azote du 30 janvier au 7 mars a conduit à une augmentation du rendement de l'ordre de 3 q.ha ${ }^{-1}$, grâce à un net accroissement de I'efficience de l'utilisation de l'azote par la culture (passant alors de 25-30 \% à 80-95\%).

Dans toutes les situations testées, quand une proportion importante de l'engrais appliqué est apportée tôt (au mois de janvier), I'accumulation $\mathrm{d}^{\prime} \mathrm{N}$ à la fin floraison est faible à cause $\mathrm{d}^{\prime}$ une trop faible valorisation de l'N apporté. Si une forte dose $d^{\prime} \mathrm{N}$ est apportée tard, le rendement peut être réduit de quelques quintaux à cause d'un mauvais état de nutrition azotée de la culture au début floraison. Ces deux constats sont cohérents avec les observations.

II serait nécessaire de comparer les stratégies de fertilisation azotée recommandées par le modèle dans des situations avec gros et petits colzas à la reprise de végétation.

\section{Conclusion}

Les premiers résultats d'évaluation et de comparaison de stratégies de fertilisation azotée sont encourageants. II est bien sûr nécessaire de les confirmer par une évaluation du modèle dans une plus large gamme de situations culturales, permettant ainsi d'appréhender son domaine de validation. Cependant, il semble déjà que ce modèle permette de raisonner la fertilisation azotée et en particulier le fractionnement de la dose $d^{\prime} \mathrm{N}$ à apporter au printemps en vue de maximiser le profit, d'optimiser la qualité des graines et de réduire les nuisances environnementales. En effet, le modèle
Azodyn-colza permet de simuler correctement les effets de l'avancement du semis, la variabilité des coefficients d'utilisation de l'azote au cours du temps, la contribution des feuilles tombées à la nutrition azotée de la culture, les effets de la fertilisation azotée sur la teneur en huile et sur le reliquat $\mathrm{d}^{\prime} \mathrm{N}$ minéral dans le sol à la récolte. Par ailleurs, sa conception ayant été réalisée en étroite collaboration avec ses futurs utilisateurs, il nous semble que les chances que ce modèle soit utilisable et utilisé dans l'avenir sont accrues.

Plusieurs autres types d'utilisation sont d'ores et déjà envisagés par le CETIOM. Le couplage du modèle avec de larges bases de données sol et climat permettrait de prévoir le rendement dans la plupart des zones de culture en France, en identifiant les limitations liées à la disponibilité de l'eau ou de l'azote [37]. L'utilisation du modèle à l'échelle d'un territoire pourrait permettre de raisonner le couple variété - fertilisation azotée, en fonction des caractéristiques des sols et des climats, en vue de maximiser la qualité des graines et le rendement des parcelles d'un organisme de collecte.

\section{RÉFÉRENCES}

1. DEJOUX JF, MEYNARD JM, REAU R, ROCHE R, SAULAS P. Évaluation of environmentallyfriendly crop management systems based on very early sowing dates for winter oilseed rape in France. Agronomie 2003 ; 23(8) : 725-36.

2. REAU R, JUNG L, WAGNER $D$. Les bases de la fertilisation azotée prévisionnelle: prévoir la dose d'azote à apporter au printemps. Oléoscope $1997 ; 20: 29$-36.

3. LIMAUX F, RECOUS S, MEYNARD JM, GUCKERT A. Relationship between rate of crop growth at date of fertiliser $\mathrm{N}$ application and fate of fertiliser $\mathrm{N}$ applied to winter wheat. Plant Soil $1999 ; 214$ : 49-59. 
4. LOYCE C, RELLIER JP, MEYNARD JM. Management planning for winter wheat with multiple objectives (2): ethanol-wheat production. Agric Syst 2002 ; 72(1) : 33-57.

5. ROSSING WAH, MEYNARD JM, VAN ITTERSUM MK. Model-based explorations to support development of sustainable farming systems: case studies from France and The Netherlands. Eur J Agr 1997 ; 7 : 271-83.

6. COLNENNE C. Les besoins en azote du colza $d$ 'hiver, courbe critique et effets de carences temporaires sur l'élaboration du rendement. Thèse de Doctorat, INAPG, Paris, 1999.

7. BHATTY RS. Influence of nitrogen fertilization on the yield, protein and oil content of two varieties of rape. Can J Plant Sci 1964; 44 : 215-7.

8. REAU R, CHAMPOLIVIER L. Augmenter la richesse en huile du tournesol par les conduites de la culture : quel enjeu régionaux. Oléoscope $2004 ; 76: 23-7$.

9. TAYLOR AJ, SMITH C], WILSON IB. Effect of irrigation and nitrogen fertilizer on yield, oil content, nitrogen accumulation and water use of canola. Fertilizer Research 1991 ; 29 : 249-60.

10. ZHAO F, EVANS E, BILSBORROW P, KEITH SEYERS I. Influence of sulphur and nitrogen on seed yield and quality of low glucosinolate oilseed rape. / Sci Food Agric 1993 ; 63 : 29-37.

11. LETERME P. Modélisation de la croissance et de la production des siliques chez le colza d'hiver ; application à l'interprétation de résultats de rendement. Thèse de Docteur-Ingénieur, INAPG, Paris, 112 p, 1985

12. HUSSON F, WALLACH D, VANDEPUTTE B. Evaluation of CECOL, a model of winter-rape (Brassica napus L.). Eur J Agr 1998 ; 8 : 205-14.

13. GABRIELLE $B$, DENOROY $P$, GOSSE G, JUSTES E, ANDERSEN MN. Development, calibration and test of CERES-type model for winter oilseed rape. Field Crops Res 1998; 57 : 95-111.

14. JEUFFROY MH, RECOUS S. Azodyn : a simple modèle simulating the date of nitrogen deficiency for decision support in wheat fertilisation. Eur / Agr 1999 ; 10 : 129-44.

15. JEUFFROY MH, BOUCHARD C, MEYNARD JM, RECOUS S. Azodyn: a tool to adapt $\mathrm{N}$-fertilisation strategies to farmers'objectives. $11^{\text {th }} \mathrm{N}$ Workshop, Reims, sept 2001, pp 465 466.
16. MEYNARD JM, CERF M, GUICHARD L, JEUFFROY MH, MAKOWSKI D. Nitrogen, Decision Support and Environmental Management. Agronomie 2002 ; 22 : 817-29.

17. DAVID C, JEUFFROY MH, LAURENT $F$, MANGIN M, MEYNARD JM. The assessment of a decision-making tool for managing nitrogen fertilization of organic winter wheat. Eur / Agr $2005 ; 23: 225-42$

18. MAILLET I. Contribution à la modélisation du nombre d'inflorescences produites par le colza d'hiver (Brassica napus L. var. oleifera). Thèse de Docteur de l'INAPG, Paris, 1992.

19. TAYO TO, MORGAN DG. Quantitative analysis of the growth, development and distribution of flowers and pods in oil-seed rape (Brasssica napus L.). J Agr Sc Cambridge 1975 ; 85 : 10310.

20. JEUFFROY MH, GIRARD ML, BARRÉ C. Qualité du blé tendre : comprendre et prévoir la teneur en protéines des grains. Perspect agric 2000 . $261: 24-31$.

21. LARMURE A, MUNIER-JOLAIN NG. A crop model component simulating $\mathrm{N}$ partitioning during seed filling in pea. Field Crops Res 2004; 85 : 135-48.

22. DEJOUX JF. Évaluation agronomique environnementale et économique d'itinéraires techniques du colza d'hiver en semis très précoces. Thèse de Doctorat INA P-G, 1999.

23. LEMAIRE G, VAN OOSTEROM E, SHEEHY J, JEUFFROY MH, MASSIGNAM A, ROSSATO L. IS crop $\mathrm{N}$ demand more closely related to dry matter accumulation or leaf area expansion during vegetative growth? Field Crops Res $2006 ; 100: 91-106$.

24. VOGRINCIC C. Mise au point et évaluation d'un outil de gestion de l'azote sur colza : AZODYN Colza. Mémoire de fin d'études ENITA, 2000.

25. JUSTES E, DENOROY P, GABRIELLE B, GOSSE G Effect of crop nitrogen status and temperature on the radiation use efficiency of winter oilseed rape. Eur J Agr 2000 ; 13 : 165-77.

26. DEVIENNE-BARRET F, JUSTES E, MACHET JM, MARY B. Integrated control of nitrate uptake by crop growth rate and soil nitrate availability under field conditions. Ann Bot (Lond) 2000 ; 86 : 995-1005.
27. COLNENNE C, MEYNARD JM, REAU R, JUSTES E, MERRIEN A. Determination of a critical nitrogen dilution curve for winter oilseed rape. Ann Bot (Lond) 1998 ; 81 : 311-7.

28. DEJOUX JF. Recous S, Meynard JM, Trinsoutrot I, Leterme Ph. The fate of nitrogen from winterfrozen rapeseed leaves: mineralization, fluxes to the environment and uptake by rape seed crop in spring. Plant Soil $2000 ; 218: 257-72$.

29. FAURE $S$. Etude de l'absorption du nitrate chez Brassica napus L. : Evolution de l'activité des transporteurs et de la transcription des gènes NRT1 et NRT2 en réponse à une privation en NO3-, évaluation de leur rôle sur le cycle de culture. Thèse de l'université de Caen, 2000.

30. COLNENNE C, MEYNARD JM, ROCHE R, REAU R. Effects of nitrogen deficiencies on autumnal growth of oilseed rape. Eur / Agr $2002 ; 17(1): 11-28$.

31. JEUFFROY MH, COLNENNE C, REAU R. Nitrogen nutrition and rapeseed seed number. Proc. $11^{\text {th }}$ International Rapeseed congress. Copenhagen Danemark, 2003.

32. MACHET JM, DUBRULLE P, LOUIS P. AZOBIL : a computer program for fertilizer $\mathrm{N}$ recommandations based on a predictive balance sheet method. In : Proceedings of the 1st Congress of the European Society for Agronomy. Paris : ed A. Scaife, $1990: 21-2$.

33. BURNS IG. Equations to predict the leaching of nitrate uniformly incorporated to a known depth or uniformly distributed throughout a soil profile. J Agric Sci Camb 1976 ; 86 : 305-13.

34. SAULAS L. Azodyn Colza: Mise au point et évaluation du module simulant la teneur en huile et le rendement. Rapport de fin d'étude d'ingénieur ISAB, 2003.

35. VALANTIN-MORISON M, JEUFFROY MH, SAULAS L, CHAMPOLIVIER L. Azodyn-rape : a simple model for decision support in rapeseed nitrogen fertilisation. 11th International rapeseed congress, Copenhaguen (Denmark), 2003/07/6-10.

36. VALANTIN-MORISON M, JEUFFROY MH, CHAMPOLIVIER L. Evaluation and sensitivity analysis of Azodyn-rape, a simple model for decision support in rapeseed nitrogen 11th Congress of European Society of Agronomy, Copenhaguen (Denmark), 2004/07/12-16.

37. CHAMPEIL A. Rapport final du projet modèle CAzoPot. Rapport interne. CETIOM, 2006. 